\title{
First aid for acute lung inflammation
}

Acute inflammation of the lung tissue or acute pneumonia (AP) is one of the oldest nosologies known to medicine since the first description by Hippocrates. The severe course of the disease, the frequent development of complications and the high mortality rate have created a halo of fear around it for more than two and a half millennia.

However, during this long period, humanity did not sit idly by. Since the study of how to care for such patients was limited by scant scientific information and modest opportunities to obtain it, constant empirical research was conducted. Of course, such research by trial and error could not pass without tragic situations.

One of the most famous tragedies related to the treatment of AP and made public was the death of the first President of the United States of America, George Washington. According to the accounts that have come down to our time, his death was not without the impact of medical procedures. ${ }^{1}$ Signs of a severe cold with fever, chest pain and difficulty breathing were most likely associated with AP, although an accurate diagnosis was never established. A group of invited doctors performed bloodletting, which at that time was widely used in such situations. Within a short time, more than half of the patient's circulating blood was released. Of course, this was done with the best intentions, although at present even paramedics know that such a volume of blood loss is incompatible with life.

Despite the lack of scientific justification and objective arguments, the centuries-old experience of medicine allows us to note the general trend in the development of first aid methods for AP. Without being able to scientifically study the problem and test various procedures, ancient medicine was able to determine exclusively empirically the importance of reducing the load of blood on the pulmonary vessels in a critical situation. In fact, the only assessment of these efforts remained the results of treatment.

For example, one of the methods of providing first aid to patients with AR, which was quite widely used in the past, was the abovementioned bloodletting. ${ }^{2}$ The very principle of this procedure is to quickly reduce the volume of circulating blood and reduce its return to the small circle of blood circulation, unloading the lungs. The blood released during the procedure was irretrievably lost to the patient's body. Despite the lack of scientific justification and strict rules of application, this method of treatment was popular for several centuries until the last century, which indirectly indicates its clinical effectiveness. It's unlikely that this technique could have remained in demand for such a long time if it hadn't brought some notable benefits, isn't it?

The category of procedures that have passed the test of time in patients with AP includes methods for directly reducing the volume of circulating blood by extracting it outside and methods for redistributing it inside the body. Methods of the first group include bloodletting and hirudotherapy. ${ }^{2,3}$ The methods of the second group include cupping therapy and body cooling..$^{4-6}$ Over the past many decades, all these methods have been excluded from the arsenal of official medicine. The use of oxygen therapy dates back a little more than a hundred years, ${ }^{7}$ but recently the supply of oxygen is the most common type of first aid in clinics in developed countries. ${ }^{8}$ In the current pandemic, in patients with COVID-19 pneumonia, oxygen therapy remains the main and almost the only method of first aid, despite the fact that there
Volume 8 Issue 3 - 202।

\author{
Igor Klepikov \\ Igor Klepikov Professor, Department of Pediatric surgery, \\ retired, USSR, USA
}

Correspondence: Igor Klepikov, Department of Pediatric surgery, retired, Igor Klepikov Professor, Department of Pediatric surgery, retired, USSR, USA, Tel + I-206-920-9643, Emailiklepikov@yahoo.com

Received: July II, 202I | Published:September 09, 202 I

is evidence that the level of oxygenation does not affect the results of treatment. ${ }^{9,10}$

A fundamental change in the principles of first aid for AP occurred in the middle of the last century, when antibiotics were introduced into medical practice. The first results of the sterilizing effect of the new drugs gave the impression that a universal treatment for inflammatory processes, including in patients with AP, was found. Previous methods of helping with inflammatory processes of the lungs, together with centuries of experience, were discarded as unnecessary. Time has long shown what the price of this illusion is, but the understanding of the self-deception that has occurred has not yet been widely accepted. The suggestive effect of the initial period of antibacterial therapy had, contrary to logic and common sense, a long-term negative didactic effect. Currently, the existing concepts of AP are not consistent with the facts and fundamentals of medical knowledge, creating a serious barrier to solving this problem.

First, antibiotics are a means of suppressing the bacterial factor, but do not have a direct effect on the inflammatory process. In the case of successful action of these drugs, stopping the development and elimination of inflammation completely falls on the patient's own strength. Specialists are well aware of this feature of etiotropic treatment, but today, by analogy with existing templates, there is an active search for antiviral agents, while patients with viral pneumonia show high mortality. It should be added that, in contrast to the extracellular activity of bacteria, viruses begin to manifest themselves by "hiding" inside the cell.

Second, attempts have been made to prove the importance of antibiotics as first aid for patients with AP. To this end, the effectiveness of antibacterial therapy was studied, depending on the speed of its use after diagnosis. However, the representative material of such studies did not reveal any significant differences..$^{11,12}$ 
Third, against the background of the growth of antibiotic-resistant strains and the decline in the effectiveness of drugs, attempts have been made for many years to conduct long-term courses of treatment. The desire for such "sterilization" of patients is contrary to the biological foundations of nature, which clearly shows the illusory nature of such efforts. In recent years, the tactics of antibacterial therapy have suddenly and insufficiently reasoned changed with the recommendation of short (up to 3-5 days) courses of AP treatment, but this did not affect the results. ${ }^{13-16}$

Despite the fact that the leading role of antibiotics in the treatment of AP has long caused dissatisfaction with the final results, and various tactics of their use do not bring success, the commitment to the old dogmas remains tragically unshakeable. In the current conditions with a large number of cases of COVID-19 pneumonia, when the use of antibiotics makes neither logic nor sense, professional recommendations to continue prescribing them to these patients are surprising. ${ }^{17}$ Moreover, clinical materials show that in this situation, the old schemes of providing medical care are not limited to recommendations. Patients with coronavirus pneumonia in the presence of a bacterial infection only in isolated cases receive antibiotics in more than $70-80$ percent. ${ }^{18-20}$ These figures reflect the confusion in the ranks of professionals and the state of stagnation in solving the problem.
Fourth, the belief that the AP clinic is determined by the nature of its pathogen, which appeared with the beginning of antibacterial therapy, led to overdiagnosis of sepsis and septic shock in a group of patients with aggressive development of the disease. These complications were not in doubt even with such a lack of evidence, when in the overwhelming number of observations, blood cultures did not reveal the pathogen. ${ }^{11,14,21-23}$ The desire to confirm this point of view was accompanied by any explanations up to such paradoxical ones that sterile blood cultures are the result of an effective(!?) antibacterial therapy. ${ }^{12}$

Fifth, after many years of treating AR on the principle of "antibiotics alone", the loss of their key role by these drugs required additional assistance. One of the most acceptable methods of auxiliary support is infusion therapy (IT). Providing access to the vein and drip administration of fluids is usually one of the first procedures when a patient is hospitalized. IT was included in the standard of treatment of patients with AR by analogy with its use in other inflammatory processes, but no special studies were conducted to justify intravenous infusions in patients with AR. Replacement of fluid losses (?) and detoxification are an automatic explanation, but not a scientific argument in favor of this technique in the AP. It is enough to look at the presented scheme (Figure 1) of various inflammatory processes in order to understand in general terms the cardinal difference in the effect of IT on the focus of inflammation in the lung.

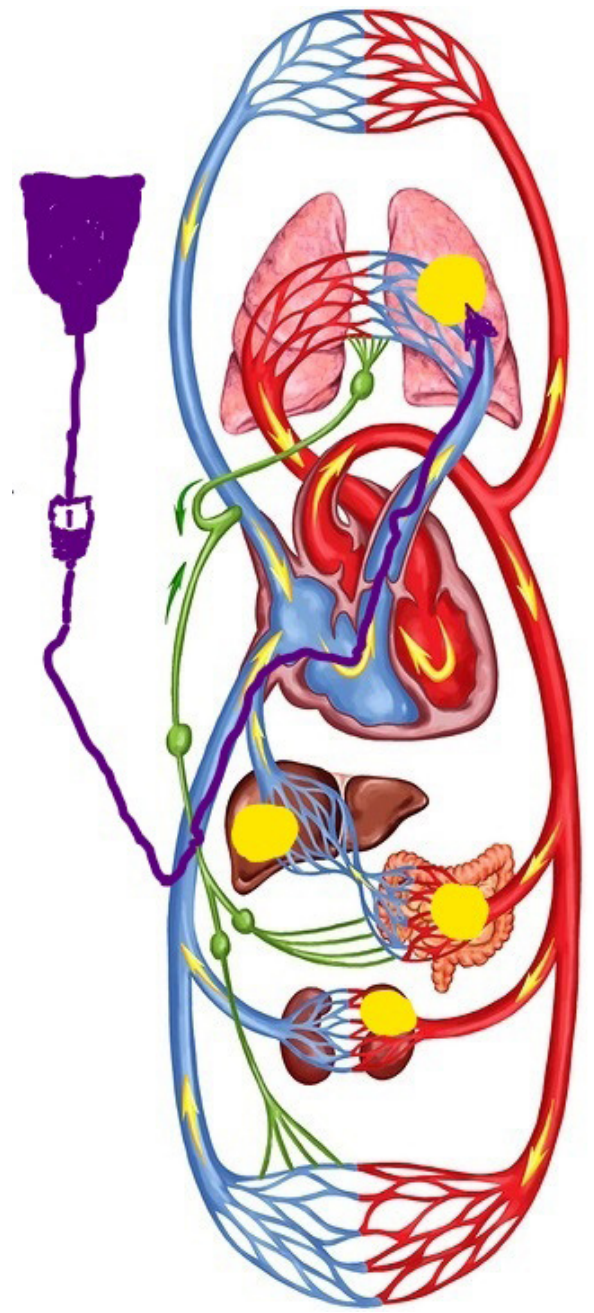

Figure I Schematic representation of the human circulatory system. The comparative value of foci of acute inflammation (yellow fields) for different departments and volumes of blood flow, depending on the possible localization. The initial route of intravenous administration of solutions (dark purple arrow). 
It should be added to the above that IT in patients with AP is an innovation of the last decades, which completely negates the previous centuries-old experience of medicine, which has a directly opposite orientation. The results of special studies have shown that the body of a patient with AP tends to unload a small circle and redistribute the circulating blood, which creates the impression of dehydration. The introduction of fluids into the venous bed in the initial period of the disease increases the load on the vessels of the lungs, stimulating edema and infiltration in the focus of inflammation. ${ }^{24-27}$

The ideology of AP, created over the past decades, and the principles of treatment resulting from it are failing. The current SARS-CoV-2 pandemic has particularly clearly demonstrated the shortcomings of existing approaches to helping these patients, destroying existing stereotypes of views. The endless spectrum of manifestations of the same type of infection from asymptomatic carrier to the most severe lung lesions has turned the dogma about the leading role of the pathogen in the inflammation of the lung tissue. The lack of effective ways to help with coronavirus pneumonia and its hasty search are a consequence of the dominant concept of the disease and suggest the continuation of the already familiar scenario.

During the pandemic, about 4 million deaths have already been registered in the world, the main cause of which is COVID-19 pneumonia. However, medicine does not yet provide effective assistance to such patients. For most specialists, the development of drugs against SARS-CoV-2 is the limit of dreams. An unprecedented search is underway for such drugs that should fill the vacant niche of etiotropic therapy for AP, but the results of these efforts remain fruitless ${ }^{28}$ Despite the endless range of clinical manifestations of coronavirus contact with the body, the pathogen remains the main cause of this problem. Now, by analogy with bacterial forms of AP, and also without objective confirmation, the severity of the patients ' condition is explained by sepsis and shock of viral etiology. ${ }^{29-31}$ At the same time, the basis of the first and further assistance to such patients during hospitalization is made up of auxiliary and supportive methods, such as oxygen supply and IT.

The current situation involuntarily forces us to recall two old wellknown truths. The first of them reads: "Sow the wind and reap the whirlwind." The excitement and joy over the discovery of antibiotics prevented a comprehensive objective assessment of this medical direction, on the initial principles of which subsequent generations were brought up. The effect of these drugs disturbed the usual balance between the body and the accompanying microflora, where the latter was subjected to prolonged suppression. The result of such an intervention can quite logically be not only the appearance of resistant strains of bacteria, but also the growth of viral pneumonia, which has been observed for at least the last two decades. ${ }^{32-34}$ Forecasts are always very difficult, but judging by the dynamics of events, it looks very much like humanity is entering a new era of viral inflammation.

The second expression, which summarizes the centuries-old experience of mankind about the role of history, says: "If we shoot at the past with a pistol, then the future will shoot at us with a cannon." An attentive and reasonable attitude to history, a rational and balanced analysis of it, a pragmatic approach to the achievements and successes of the past are the basis on which a further strategy for solving any problems should be built. If we look at the historical approach to the treatment of AP from these positions, then only one conclusion clearly suggests itself: for two and a half millennia of acquaintance with this disease, medicine has not been able to develop and offer any methods of assistance worthy of attention and application today. Is this really the case? Such a conclusion is absolutely wrong and can only be made by a person who ignores the canons of medicine and biology.
The above brief digression into the history of AP treatment and a comparison of old and modern principles of first aid for this disease, not without reason, lead to completely different conclusions. First, the focus of acute inflammation in the lung tissue, regardless of its etiology, will inevitably be accompanied by the classical signs of this process, described many centuries ago by Celsus and Galen (heat, pain, redness, swelling, and loss of function). Modern medical science does not deny these manifestations of inflammation, and a violation of the function of the affected organ is the most significant clinical sign that determines the features of various localizations.

Secondly, the development of the focus of inflammation, and not its etiology, determines the severity of functional disorders. Acute inflammation of $50-60 \%$ of the lung tissue will be more severe than $5-10 \%$, right? Therefore, etiotropic drugs are not able to help the rapid adaptation of the body and cannot be considered as first aid to such patients. The fallacy of hopes for the primary suppression of the pathogen is especially noticeable with a hyperergic inflammatory reaction and the corresponding aggressive development of AP.

Thirdly, the methods of old medicine, which were used as first aid for AP, are aimed at reducing venous return and unloading the lungs. Modern principles of saving such patients have the opposite effect. The presence of hypotension in the patient is considered as a fluid deficiency, but not as a protective reaction, and the "unwillingness" of the body to increase peripheral pressure in response to infusion is the reason for the appointment of vasopressors. At the same time, in addition to the already known materials about the restructuring of blood circulation in response to a focus of irritation in a small circle, new confirmations of this fact were obtained already with COVID-19 pneumonia, ${ }^{35}$ which did not entail changes in the treatment strategy and are evaluated only as a reason for further research. ${ }^{36}$

Currently, the entire strategy for solving the problem of AP is still completely focused on the causative agent of the disease, although the fundamental role of inflammatory transformation of lung tissues today looks more obvious than a few decades ago. Many of the previous methods of first aid for AR have been preserved in medical practice to this day, but they are not available to the overwhelming number of emergency patients. First of all, this is due to the fact that traditional medicine interprets the causes of the severity of the disease from a different angle, and the use of old methods of treatment is allowed only in alternative medicine clinics. At the official level, for example, cupping therapy is even considered as quackery. ${ }^{4}$

And yet, to discard the centuries-old experience of medicine just because it does not coincide with modern ideas about the nature of the disease, looks, to put it mildly, not a very wise decision. Especially if we take into account the fact that the current care for patients with AP has reached a dead end. Is it not better in this situation to resort to testing those methods that have been used for many centuries? Moreover, the possibilities of medical research currently allow for an objective comparative assessment of various methods of first aid and to obtain confirmation of the necessary therapeutic actions. ${ }^{37}$ This approach to solving this problem is a proven direction, which is confirmed by clinical results. ${ }^{25,26}$ The only, but very difficult obstacle on the way to achieving this goal, which must inevitably be overcome, remains a distorted conceptual idea of the nature of the disease.

\section{Acknowledgments}

None.

\section{Conflicts of interest}

None. 


\section{References}

1. George Washington.

2. Bloodletting.

3. Leech.

4. Cupping therapy.

5. Ice bath.

6. Whole body cryotherapy. In: Cryotherapy.

7. Oxygen therapy.

8. Wyatt, Jonathan P, Illingworth, et al. Oxford Handbook of Emergency Medicine. 2012.

9. OL Schjørring, TL Klitgaard, A Perner, et al. Lower or Higher Oxygenation Targets for Acute Hypoxemic Respiratory Failure. $N$ Engl $J$ Med. 2021;384(14):1301-1311.

10. PJ Young. Effect of Oxygen Therapy on Mortality in the ICU. $N$ Engl $J$ Med. 2021;384:1361-1363.

11. Liapikou E. Rosales-Mayor A. Torres. The management of severe community acquired pneumonia in the ICU. June 2014, Expert Review of Respiratory Medicine. 2014;8(3):293303.

12. Schlapbach LJ, Weiss SL, Wolf J. Reducing collateral damage from mandates for time to antibiotics in pediatric sepsis-primum non nocere. JAMA Pediatr. 2019;173:409-410.

13. Revised WHO classification and treatment of childhood pneumonia at health facilities. Geneva: World Health Organization. 2014.

14. JP. Metlay, GW Waterer, AC Long, et al. on behalf of the american thoracic society and infectious diseases society of america. "diagnosis and treatment of adults with community-acquired pneumonia. An official clinical practice guideline of the american thoracic society and infectious diseases society of america". American Journal of Respiratory and Critical Care Medicine. 2019;200(7):47-67.

15. Weiss SL, Peters MJ, Alhazzani W, et al. Surviving sepsis campaign international guidelines for the management of septic shock and sepsisassociated organ dysfunction in children. Pediatr Crit Care Med. 2020;21(2):52-106.

16. B Chang, K Grimwood. Antibiotics for childhood pneumonia - do we really know how long to treat? $N$ Engl J Med. 2020;383(1):77-79

17. BD Huttner, G Catho, JR Pano-Pardo, et al. COVID-19: don't neglect antimicrobial stewardship principles. Clin Microbiol Infect. 2020;26(7):808-810.

18. Rawson TM, Moore LSP, Zhu N, et al. Bacterial and fungal co-infection in individuals with coronavirus: A rapid review to support COVID-19 antimicrobial prescribing .Clin Infect Dis. 2020;71(9):2459-2468.

19. Beović M. Doušak J, Ferreira-Coimbra, et al. Antibiotic use in patients with COVID-19: a 'snapshot' Infectious Diseases International Research Initiative (ID-IRI) survey. $J$ Antimicrob Chemother. 2020;75(11):3386-3390.
20. Kim D, Quinn J, Pinsky B, et al. Rates of co-infection between SARSCoV-2 and other respiratory pathogens. JAMA. 2020;323(20):2085-2086.

21. GW Waterer, MW Quasney, RM. Cantor, et al. Septic Shock and Respiratory Failure in Community-acquired Pneumonia Have Different TNF Polymorphism Associations. AJRCCM. 2001;163(7):1599-1604.

22. AJ Morgan, AJ Glossop. Severe community -acquired pneumonia. BJA Education. 2016;16(5):167-172,

23. Ana Vidala, Lurdes Santos. Comorbidities impact on the prognosis of severe acute community-acquired pneumonia. Porto Biomedical Journal. 2017;2(6): 247-346

24. Klepikov Igor. "The Effect of intravenous infusion on the dynamics of acute pneumonia". EC Pulmonology and Respiratory Medicine. 2017;4.1(2017):15-20.

25. Klepikov I. Acute pneumonia. New doctrine and first treatment results. Lambert Academic. 2020.

26. Igor Klepikov. How many Pneumonias exist in nature? Generis Publishing. 2021.

27. D. A. Berlin, R. M. Gulick, F. J. Martinez. Severe Covid-19. NEJM. 2020

28. WHO Solidarity trial consortium. repurposed antiviral drugs for Covid-19 - Interim WHO Solidarity Trial Results. $N$ Engl J Med. 2021;384(6):497-511.

29. Alhazzani W. Surviving sepsis campaign: Guidelines on the management of critically ill adults with coronavirus disease 2019 (COVID-19). Crit Care Med. 2020;46(5):854-887.

30. COVID-19 Treatment Guidelines. NIH.

31. O Coz Yataco, SQ. Simpson. Coronavirus disease 2019 sepsis. A nudge toward antibiotic stewardship. Chest. 2020;158(5):1833-1834.

32. WHO Revised global burden of disease 2002 estimates. 2004.

33. Rudan I, Boschi-Pinto C, Biloglav Z, et al. Epidemiology and etiology of childhood pneumonia. Bull World Health Organ. 2008;86:408-416.

34. Ruuskanen O, Lahti E, Jennings LC, et al. Viral pneumonia. Lancet. 2011;377 (9773):1264-1275.

35. Thillai M, Patvardhan C, Swietlik EM, et al. Functional respiratory imaging identifies redistribution of pulmonary blood flow in patients with COVID-19. Thorax. 2021;76:182-184.

36. van Es J, Meijboom LJ. Functional respiratory imaging repurposed for COVID-19. Thorax. 2021;76:107.

37. Igor Klepikov. First Aid for Aggressive Forms of Acute Pneumonia". EC Pulmonology and Respiratory Medicine. 2018;72(2018):34-37. 